Citation: Y1ldız, G, Daghan, D . "Solution of the (2+1) Dimensional Breaking Soliton Equation by Using Two Different Methods". Journal of Engineering Technology and Applied Sciences 1 (1) 2016 : 13-18

\title{
SOLUTION OF THE (2+1) DIMENSIONAL BREAKING SOLITON EQUATION BY USING TWO DIFFERENT METHODS
}

\author{
Guldem Yıldız $^{a^{*}}$, Durmus Daghan ${ }^{b}$ \\ ${ }^{a}$ Department of Mathematics, Faculty of Arts and Sciences, Omer Halisdemir University, 51200 , \\ Nigde, Turkey, \\ guldem.yildiz@ohu.edu.tr*(correspondingauthor) \\ ${ }^{b}$ Department of Mathematics, Faculty of Arts and Sciences, Omer Halisdemir University, 51200, \\ Nigde, Turkey, \\ durmusdaghan@ohu.edu.tr
}

\begin{abstract}
In this study, the direct integration and homotopy perturbation method are used for the non-linear partial differential $(2+1)$ dimensional breaking soliton equation. By assigning some special values to the constants in the solutions of the $(2+1)$ dimensional breaking soliton equation, The direct integration was used for obtaining the known solution in the literature in practical and shortest way. By using the homotopy perturbation method with one iteration, it was obtained same type solution to $(2+1)$ dimensional breaking soliton equation. Similarly, same type solutions could be done in different methods such as $\left(\mathrm{G}^{\prime} / \mathrm{G}\right)$-expansion method.
\end{abstract}

Keywords: Breaking soliton equation, homotopy perturbation method, direct integration .

\section{Introduction}

Breaking soliton equations can be implemented to the model $(2+1)$ dimensional interaction of Riemann-wave and long-wave propagations in engineering and sciences. $(2+1)$ dimensional breaking soliton equation is a kind of different $(2+1)$ dimensional $\mathrm{KdV}$ extensions in the following form [1]:

$$
u_{x t}-u_{x x x y}-2 u_{x x} u_{y}-4 u_{x} u_{x y}=0
$$

where $u=u(\mathrm{x}, \mathrm{y}, \mathrm{t})$. This equation was first revealed in [2-3]. In [4] paper it was investigated $(2+1)$-dimensional breaking soliton equation (1) via the generalized tanh method. In [5] paper using computerised symbolic computation were obtained for $(2+1)$ dimensional breaking soliton equation. This equation that is given in $\mathrm{Eq}(1)$ have been investigated by many authors 
[6-18]. In this study, we use the direct integration to exact solution of the (2+1)-dimensional breaking soliton equation

In addition, we investigate same type solution for $(2+1)$ dimensional breaking soliton equation by using the homotopy perturbation method with one iteration.

$$
u_{x t}-u_{x x x y}-2 u_{x x} u_{y}-4 u_{x} u_{x y}=0
$$

where $u=u(\mathrm{x}, \mathrm{y}, \mathrm{t})$. This equation was first revealed in [2-3]. In [4] paper it was investigated $(2+1)$-dimensional breaking soliton equation (1) via the generalized tanh method. In [5] paper using computerised symbolic computation were obtained for $(2+1)$ dimensional breaking soliton equation. This equation that is given in $\mathrm{Eq}(1)$ have been investigated by many authors [6-18]. In this study, we use the direct integration to exact solution of the (2+1)-dimensional breaking soliton equation. In addition, we investigate same type solution for $(2+1)$ dimensional breaking soliton equation by using the homotopy perturbation method with one iteration.

\section{Solution of the breaking soliton equation by using direct integration}

In this section, we will present to exact solution of the $(2+1)$ dimensional breaking soliton equation [1] given in Eq (1) by using the direct integration. Seek solution of Eq (1) by taking $u(\mathrm{x}, \mathrm{y}, \mathrm{t})=\mathrm{U}(\eta), \eta=k_{1} x+k_{2} y+v t$ and transforming Eq (1) to the ordinary differential equation

$$
k_{1}^{2} k_{2} U^{(4)}-v U^{\prime \prime}+6 k_{1} k_{2} U^{\prime \prime} U^{\prime}=0
$$

where prime denotes the derivative with respect to $\eta$. Integrate Eq (2) term by term one time

$$
k_{1}^{2} k_{2} U^{(3)}-v U^{\prime}+3 k_{1} k_{2}\left(U^{\prime}\right)^{2}+c=0
$$

where $c$ is an arbitrary integration constant. For simplicity, the integration constant can be set to zero. Defining the transformation $U^{\prime}=w$ we get

$$
k_{1}^{2} k_{2} w^{\prime \prime}-v w+3 k_{1} k_{2} w^{2}=0
$$

Integrate Eq (3) term by term one time

$$
\left(w^{\prime}\right)^{2}-\frac{v}{k_{1}^{2} k_{2}} w^{2}+\frac{2}{k_{1}} w^{3}+d=0
$$

where $d$ is an arbitrary integration constant. For simplicity, the integration constant can be set to zero. Thus, we have

$$
w^{\prime}=\mp \sqrt{\frac{v}{k_{1}^{2} k_{2}} w^{2}-\frac{2}{k_{1}} w^{3}}
$$

Eq. (4) can be written as follows for plus signature (similar calculation can be done for negative signature): 


$$
\int \frac{d w}{\sqrt{\frac{v}{k_{1}^{2} k_{2}} w^{2}-\frac{2}{k_{1}} w^{3}}}=\int d \eta
$$

By the integrating right side of this equation (5), we get

$$
\int \frac{d w}{\sqrt{\frac{v}{k_{1}^{2} k_{2}} w^{2}-\frac{2}{k_{1}} w^{3}}}=\eta+e
$$

we choose integration constant $e=0$ where $e$ is a new arbitrary integration constant. In this case, the solution of the Eq. (6)

$$
w(\eta)=\frac{v}{2 k_{1} k_{2}}\left(1-\tanh ^{2}\left[\frac{\sqrt{v} \eta}{2 k_{1} \sqrt{k_{2}}}\right]\right)
$$

Finally, $w(\eta)$ is integrated. The exact solution of the $(2+1)$ Dimensional Breaking Soliton Equation given in Eq. (1) is

$$
U=\sqrt{\frac{v}{k_{2}}} \tanh \left[\frac{1}{2 k_{1}} \sqrt{\frac{v}{k_{2}}} \eta\right]
$$

\section{Homotopy perturbation method}

For the utility of the reader, we will introduce HPM [19]. The following nonlinear differential equation:

$$
A(u)-f(r)=0, r \in \Omega
$$

with boundary conditions

$B(u)-\partial u / \partial n=0, \quad r \in \Gamma$

where $A$ is a general differential operator, $B$ is a boundary operator, $f(r)$ is a known analytical function and $\Gamma$ is the boundary of the domain $\Omega$. The operator $A$ can be divided into two parts $L$ and $N$,

$L(u)+N(u)=f(r), r \in \Omega$

where $L$ is linear and $N$ is nonlinear. It was constructed a homotopy $v(r, p)=\Omega \times[0,1] \rightarrow R$ in paper [19]. $v$ satisfies

$$
H(v, p)=L(v)-L\left(u_{0}\right)+p L\left(u_{0}\right)+p[N(v)-f(r)]=0
$$


where $p \in[0,1]$ is embedding parameter and $u_{0}$ is an initial approximation of Eq. expressly we have

$$
\begin{gathered}
H(v, 0)=L(v)-L\left(u_{0}\right)=0 \\
H(v, 1)=L(v)+N(v)-f(r)=0
\end{gathered}
$$

where $L(v)-L\left(u_{0}\right)$ and $A(u)-f(r)$ are called homotopic in topology. According to the homotopy perturbation technique, we can be written the solution Eq (9) as a power series in $p$ small parameter:

$$
v=v_{0}+p v_{1}+p^{2} v_{2}+p^{3} v_{3}+\cdots
$$

Setting $p=1$ results in the approximate solution of Eq. (8)

$$
u=\lim _{p \rightarrow 1} v=v_{0}+v_{1}+v_{2}+\cdots
$$

\section{Application of homotopy perturbation method for $(2+1)$ dimensional breaking soliton equation}

It is well known that the homotopy perturbation method in [18-22], we use for Eq. (10). We compose a homotopy in the form

$$
w^{\prime \prime}-\frac{v}{k_{1}^{2} k_{2}} w+\frac{3}{k_{1}} p w^{2}=0
$$

where $p \in[0,1]$. In Eq.(4) there are two observations to be considered; the first observation is that for $p=0$, Eq.(10) becomes a linear equation. The second observation is that for $p=1$; it becomes the original nonlinear one. Due to the homotopy perturbation method, the solution of Eq.(10) can be expressed in a series of $p$ :

$$
\begin{aligned}
& w=w_{0}+p w_{1} \\
& \frac{v}{k_{1}^{2} k_{2}}=h^{2}+p h_{1}
\end{aligned}
$$

Substituting Eqs (11) and (12) into Eq. (10) and equating coefficients of like powers of $p$ yields a linear equation:

$$
w_{0}^{\prime \prime}-h^{2} w_{0}=0
$$

Solving of Eq. (13)

$$
w_{0}(\eta)=c_{1} e^{h \eta}+c_{2} e^{-h \eta}
$$


This solution is determinant for the $w_{0}(0)=x, w_{0}^{\prime}(0)=y$ initial conditions, where $y=0$. We get

$x=\frac{1}{2} \sec h^{3}(\mathrm{~h} \eta)$. Therefore, we obtain

$$
w_{0}(\eta)=\frac{1}{2} \sec h^{2}(\mathrm{~h} \eta)
$$

Finally, $w_{0}(\eta)$ is integrated. Approximate solution of the breaking soliton equation given in Eq. (1) is

$$
U=\frac{k_{1}}{2} \sqrt{\frac{k_{2}}{v}} \tanh \left[\frac{1}{k_{1}} \sqrt{\frac{v}{k_{2}}} \eta\right]
$$

\section{Conclusions}

In this study, the direct integration was used to obtain exact solution of the $(2+1)$ dimensional breaking soliton equation. On the other hand, the homotopy perturbation method was used to obtain the perturbative solution of the $(2+1)$ dimensional breaking soliton equation. We compared the results obtained with both methods. Obtained results from direct integration and the homotopy perturbation method are the same with tanh solutions. Furthermore, obtained solution from the direct integration is identical with solution in the article of Arbabi and Najafi (2016) [1].

\section{References}

[1] Arbabi, Somayeh, Mohammad Najafi, "Soliton Solutions of Nonlinear Evolution Equations in Mathematical Physics.”Optik-International Journal for Light and Electron Optics 127.10 (2016): 4270-4274.

[2] Calogero, F., A. Degasperis, "Nonlinear Evolution Equations Solvable by The Inverse Spectral Transform. - I.” Il Nuovo Cimento B (1971-1996) 32.2 (1976): 201-242.

[3] Calogero, F., A. Degasperis, "Nonlinear Evolution Equations Solvable by The Inverse Spectral Transform. - II.” Il Nuovo Cimento B (1971-1996) 39.1 (1977): 1-54.

[4] Tian, Bo, Keyi Zhao, Yi-Tian Gao, "Symbolic Computation in Engineering: Application to A Breaking Soliton Equation." International journal of engineering science 35.10-11 (1997): 1081-1083.

[5] Yan, Zhen-Ya, Hong-Qing Zhang, "Constructing Families of Soliton-Like Solutions to A $(2+1)$-Dimensional Breaking Soliton Equation Using Symbolic Computation.” Computers \& Mathematics with Applications 44.10 (2002): 1439-1444.

[6] Geng, Xianguo, Cewen Cao, "Explicit Solutions of The 2+ 1-Dimensional Breaking Soliton Equation.” Chaos, Solitons \& Fractals 22.3 (2004): 683-691.

[7] Mei, Jian-qin, Hong-qing Zhang, "New types of exact solutions for a breaking soliton equation." Chaos, Solitons \& Fractals 20.4 (2004): 771-777. 
[8] Zhang, Sheng, "New Exact Non-Traveling Wave and Coefficient Function Solutions of The (2+ 1)-Dimensional Breaking Soliton Equations." Physics Letters A 368.6 (2007): 470-475.

[9] Zhang, Sheng, "A Generalized New Auxiliary Equation Method and Its Application to The (2+ 1)-Dimensional Breaking Soliton Equations." Applied mathematics and computation 190.1 (2007): 510-516.

[10] Ma, Song-Hua, Jian-Ping Fang, Chun-Long Zheng, "New Exact Solutions of The $(2+1)-$ Dimensional Breaking Soliton System Via An Extended Mapping Method." Chaos, Solitons \& Fractals 40.1 (2009): 210-214.

[11] Tao, Zhao-Ling, "Solving the Breaking Soliton Equation By He's Variational Method."Computers \& Mathematics with Applications 58.11 (2009): 2395-2397.

[12] Da-Quan, Xian, "Symmetry Reduction and New Non-Traveling Wave Solutions Of (2+ 1)-Dimensional Breaking Soliton Equation." Communications in Nonlinear Science and Numerical Simulation 15.8 (2010): 2061-2065.

[13] Zhao, Zhanhui, Zhengde Dai, Gui Mu, "The Breather-Type and Periodic-Type Soliton Solutions for the $(2+1)$-Dimensional Breaking Soliton Equation." Computers \& Mathematics with Applications 61.8 (2011): 2048-2052.

[14] Y1ldız, Guldem, Durmus Daghan, "Solution of the (2+1) Dimensional Breaking Soliton Equation by Using Two Different Methods." 11-16.

[15] Zayed, Elsayed, Mahmoud Abdelaziz, Mushrifa Elmalky, "Enhanced (G'/G)-Expansion Method and Applications to the $(2+1)$ D Typical Breaking Soliton and Burgers Equations." Journal of Advanced Mathematical Studies 4.2 (2011): 109-123.

[16] M.T. Darvishi, M. Najafi, "Some Exact Solutions of the (2+1)-Dimensional Break-Ing Soliton Equation Using The Three-Wave Method”, World Acad. Sci. Eng.Technol., 2011, 55, 919-922.

[17] Darvishi, Mohammad. Taghi, Mohammad Najafi, "Some Exact Solutions of the (2+1)Dimensional Breaking Soliton Equation Using The Three-Wave Method." International Journal of Computational and Mathematical Sciences 6.1 (2012): 13-16.

[18] Xu, Gui-qiong, "Integrability of A (2+ 1)-Dimensional Generalized Breaking Soliton Equation.” Applied Mathematics Letters 50 (2015): 16-22.

[19] He, Ji-Huan, "Homotopy Perturbation Technique."Computer methods in applied mechanics and engineering 178.3 (1999): 257-262.

[20] He, Ji-Huan, "A Coupling Method of a Homotopy Technique and A Perturbation Technique for Non-Linear Problems.” International journal of non-linear mechanics 35.1 (2000): 37-43.

[21] He, Ji-Huan, "Homotopy perturbation method for Bifurcation of Nonlinear Problems." International Journal of Nonlinear Sciences and Numerical Simulation 6.2 (2005): 207208.

[22] El-Shahed, Moustafa, “Application of He's Homotopy perturbation method to Volterra's Integro-Differential Equation." International Journal of Nonlinear Sciences and Numerical Simulation 6.2 (2005): 163-168. 